\title{
CONTROL MECÁNICO DEL ÁRBOL NO NATIVO LIGUSTRUM LUCIDUM (OLEACEAE): SUPERVIVENCIA, REGENERACIÓN Y COSTOS
}

\author{
MECHANICAL CONTROL OF THE NON-NATIVE TREE LIGUSTRUM LUCIDUM. \\ SURVIVAL, REGENERATION, AND COST
}

\author{
Tatiana Alejandra Valfré-Giorello $0^{1,2^{*}}\left(\mathbb{D}\right.$, Romina Cecilia Torres ${ }^{1,2} \mathbb{D}$, Fernando \\ Rafael Barri'1,3 y Daniel Renison ${ }^{1,2}$ (D)
}

\begin{abstract}
1. Centro de Ecología y Recursos Naturales Renovables-Dr. Ricardo Luti.

2. Instituto de Investigaciones Biológicas y Tecnológicas (IIByT, CONICET-UNC).

3. Instituto de Diversidad y Ecología Animal (IDEA, CONICETUNC).

\section{*tativalfre@gmail.com}

\section{Citar este artículo}

VALFRÉ-GIORELLO, T. A., R. C. TORRES, F. R. BARRI \& D. RENISON. 2019. Control mecánico del árbol no nativo Ligustrum lucidum (Oleaceae): supervivencia, regeneración y costos. Bol. Soc. Argent. Bot. 54: 93-104.

DOI: http://dx.doi. org/10.31055/1851.2372.v54. n1.23588

Recibido: 11 Junio 2018

Aceptado: 16 Noviembre 2018

Publicado: 28 Marzo 2019

Editor: Omar Varela (D)

ISSN versión impresa 0373-580X ISSN versión on-line 1851-2372

\section{SUMMARY}

Background and Aims: There is an increasing demand of cost-benefit studies for the control of invasive non-native species. We assessed the efficiency of the application of mechanical control treatments on the non-native species Ligustrum lucidum, through resprouting and seedling regeneration, integrating cost and effort.

M\&M: The study involved 120 trees, to which we applied the following treatments: 1) control group, uncut trees, 2) felling, 3) felling and covering the stump with black polyethylene. Additionally, a half of the trees were selected in forest physiognomy and a half in shrubland physiognomy. We removed all seedlings smaller than $50 \mathrm{~cm}$ tall around each treated tree.

Results: After 12 months, and two treatment applications, survival for the control group, felling, and felling with polyethylene cover was $100 \%, 95 \%$ and $68 \%$, respectively. Resprout height was $78 \%$ and $95 \%$ lower under felling and felling with polyethylene cover treatments, respectively, than in the control group. Vegetation physiognomy had no effect on survival and resprout growth, while seedling regeneration was $300 \%$ higher in forest than in shrubland. The cost of application of felling with polyethylene cover was $150 \%$ higher than the cost of applying of felling only.

Conclusions: To control L. Iucidum, we recommend felling with polyethylene cover because the higher cost and effort is compensated by its greater efficacy in the reduction of survival and height. We recommend the manual removal of seedlings mainly in sites with a shrubland physiognomy and a low cover of adults of L. lucidum.

\section{KeY Words}

Black polyethylene, efficiency, felling, mechanical control, non-native woody species, resprout, seedling.

\section{RESUMEN}

Introducción y objetivos: Existe una creciente demanda de estudios que evalúen el costo-beneficio de la aplicación de técnicas de control de especies no nativas. Evaluamos la eficiencia de la aplicación de dos tratamientos de control mecánico sobre la especie no nativa Ligustrum lucidum, a través de la regeneración por rebrote y por semilla, integrando costos y esfuerzo.

M\&M: Asignamos 120 árboles a tres grupos de tratamientos: 1) testigo, sin tala ni aplicación de polietileno; 2) tala, y 3) tala con aplicación de polietileno sobre el tocón. Seleccionamos 60 árboles en fisonomía de bosque y 60 en fisonomía de matorral. Removimos manualmente todos los plantines menores a $50 \mathrm{~cm}$ alrededor de cada árbol tratado.

Resultados: Transcurridos 12 meses y dos aplicaciones de tratamientos, la supervivencia de los testigos, tala y tala con polietileno fue $100 \%, 95 \%$ y $68 \%$, respectivamente. La altura de los rebrotes en el tratamiento de tala y en el de tala con polietileno fue $78 \%$ y $95 \%$ menor respectivamente, comparado con los individuos testigos. La fisonomía del sitio solo influyó sobre la densidad de plantines, siendo $300 \%$ mayor en bosque que en matorrales. El costo del tratamiento tala con polietileno fue $150 \%$ mayor al costo del tratamiento tala, pero fue compensado por la mayor eficacia para reducir la supervivencia y la altura de rebrotes.

Conclusiones: Debido a la mayor eficiencia, recomendamos la aplicación del tratamiento de tala con polietileno. Sugerimos remover manualmente los plantines en matorrales o sitos con baja cobertura de adultos de L. Iucidum.

\section{Palabras clave}

Control mecánico, eficiencia, especies leñosas no nativas, plantines, polietileno negro, rebrotes, tala. 


\section{INTRODUCCIÓN}

Está ampliamente documentado que las invasiones biológicas son uno de los problemas más graves para la conservación de la biodiversidad y la provisión de servicios ambientales (Pejchar \& Mooney, 2009; Simberloff et al., 2013; Pyšek \& Richardson, 2010). Las plantas no nativas, por ejemplo, generan profundas transformaciones en las comunidades donde invaden, tales como alteraciones en los regímenes de fuego, los ciclos de nutrientes, la hidrología, y pueden afectar la abundancia y supervivencia de las plantas nativas (Pyšek \& Richardson, 2010). Estas transformaciones están causando además, considerables impactos económicos (Pimentel et al., 2001; Epanchin-Niell \& Hastings, 2010; Pyšek \& Richardson, 2010; Kettenring \& Adams, 2011) por lo cual el control de las plantas no nativas invasoras se ha transformado en una necesidad urgente a nivel global y una parte fundamental en muchas iniciativas de restauración de los ecosistemas (Rejmánek \& Pitcairn, 2002; Zalba \& Villamil, 2002).

En la actualidad hay una fuerte demanda de estudios que se enfoquen en determinar aquellas técnicas de control de plantas no nativas que sean más eficientes en términos de costo-beneficio, y que proporcionen el menor impacto negativo posible en el ecosistema nativo (Kettenring \& Adams, 2011). Si bien una de las técnicas más difundidas para el control de plantas no nativas es la aplicación de herbicidas (Tu et al., 2001), muchas veces su uso es rechazado por la sociedad (McCarthy et al., 2011) debido a posibles consecuencias sobre la salud humana, el ambiente, y por la creciente elevación de precios de estos productos (Little et al., 2006).

Otras de las técnicas ampliamente usadas para el control de plantas no nativas, en especial para especies leñosas, son los tratamientos mecánicos de tala y extracción manual de individuos. Si bien los resultados de la aplicación de estas técnicas suelen ser variables, ya que muchas especies pueden rebrotar luego de la tala, tienen la ventaja de generar menos impactos negativos en el ambiente nativo y su eficacia puede incrementarse mediante variaciones en la aplicación del corte (Kettenring \& Adams, 2011). En este sentido, una forma de disminuir el crecimiento de los rebrotes luego de la tala es mediante la aplicación de barreras físicas que impidan la incidencia de la luz sobre estructuras de regeneración del individuo, como la aplicación de polietileno negro sobre tocones o ramas (Mowat, 1981; Patch et al., 1998), debido a que en condiciones de baja accesibilidad a la luz se producen tejidos débiles y etiolados (Taiz \& Zeiger, 2010).

A su vez, la eficacia de estas técnicas puede incrementarse aprovechando ciertas características del ambiente. Por ejemplo, los árboles que están inmersos en un parche de bosque, al tener menos acceso a la luz, nutrientes y agua debido a la competencia suelen tener menor capacidad de fotosintetizar e invierten sus reservas en el crecimiento en altura en búsqueda de luz (Packham et al., 1992), lo cual al momento de sufrir un disturbio suelen presentar menor capacidad de rebrote (Kays \& Canham, 1991; King et al., 2005). De esta manera, la aplicación de tratamientos de control mecánico en árboles inmersos en un parche de bosque puede resultar más eficaz en la reducción de la capacidad de rebrote que en árboles que se encuentran en sitios abiertos. Asimismo, el tamaño de un individuo muchas veces es un indicador de la disponibilidad de reservas y, en consecuencia, de la capacidad para rebrotar luego de un disturbio (Gurvich et al., 2005; Herrero et al., 2016), por lo cual la eficacia del control mecánico puede aumentar si se aplica en individuos jóvenes.

Otro aspecto importante a considerar luego de los tratamientos de control es la regeneración de plantines del invasor (Sanhueza \& Zalba, 2012). Los cambios ambientales producidos por la remoción mecánica de la vegetación suelen influir en las primeras etapas de la regeneración de las especies invasoras, porque afectan la disponibilidad de recursos y la competencia (Davis et al., 2000). En este sentido, los cambios en la cobertura del dosel dados por la aplicación de la tala pueden afectar la incidencia de luz, la temperatura y humedad que recibe el suelo, y de esta manera influir en la germinación, supervivencia y crecimiento de plantines (Burnham \& Lee, 2010).

Por otra parte, muchos estudios sobre especies invasoras se han centrado en aspectos teóricos como la magnitud e impacto de las invasiones, los cuales si bien son fundamentales para el diseño de planes de manejo, carecen del enfoque práctico necesario para el control a campo (Kettenring \& Adams, 2011). Para que los resultados de los estudios sobre control de especies no nativas puedan ser 


\section{T. A. Valfré-Giorello et al. - Control mecánico de Ligustrum lucidum}

aplicados es necesario evaluar tanto la eficacia de las técnicas de control como sus aspectos logísticos, en relación a los costos en términos de tiempo (horas de trabajo), salario de los operarios, insumos y equipamiento (Rejmánek \& Pitcairn, 2002). Esto es fundamental para que las técnicas de control puedan ser implementadas de manera extensiva por las personas encargadas de realizar el manejo (Kettenring \& Adams, 2011).

En el presente estudio nos enfocamos en Ligustrum lucidum W. T. Aiton (Oleaceae), una especie leñosa originaria de Asia, invasora en diversos ecosistemas del mundo (Richardson \& Rejmánek, 2011), así como pastizales, bosques y selvas en Argentina (Zalba \& Villamil, 2002; Aragón \& Groom, 2003; Lewis et al., 2004; Hoyos et al., 2010). Ligustrum lucidum posee varias características que la hacen difícil de controlar: rápido crecimiento, fructificación abundante con dispersión ornitocora (Montaldo, 1993; Aragón \& Groom, 2003; Ferreras et al., 2008), reproducción por semilla y reproducción vegetativa a través del rebrote de raíz (Lichstein et al., 2004). Además, tiene la capacidad de rebrotar luego de un corte (Starr et al., 2003; Lichstein et al., 2004). Posiblemente estas particularidades le han permitido invadir áreas con distintas historias de uso, ocupando desde sitios abiertos afectados por disturbios (Gavier-Pizarro \& Bucher, 2004; Hoyos et al., 2010) hasta rodales sombreados y sin disturbios (Hoyos et al., 2010; Aragón et al., 2014).

Con el fin de contribuir al control y manejo de especies no nativas en el Chaco Serrano, en el presente estudio evaluamos el éxito de la aplicación de técnicas de control mecánico en el desempeño de L. lucidum. Específicamente nos propusimos: (1) evaluar la supervivencia y altura de rebrote de $L$. lucidum luego de la aplicación de dos tratamientos de control mecánico: tala del fuste, y tala del fuste con recubrimiento de polietileno negro; (2) evaluar la regeneración por semilla de plantines de $L$. lucidum en parcelas sometidas a dichos tratamientos de control mecánico de los individuos adultos; (3) comparar la respuesta de L. lucidum a los tratamientos de control mecánico según la fisonomía de la vegetación circundante; (4) estimar la eficiencia de los tratamientos de control mecánico, integrando los costos y esfuerzo a los resultados.

Como hipótesis planteamos que, en la fisonomía de bosque la eficacia de la tala es mayor que en los matorrales debido a que los individuos han tenido menor acceso a los distintos recursos, como luz, agua y nutrientes como resultado de la competencia, lo cual reduce la capacidad de rebrote luego de un disturbio (Kays \& Canham, 1991; King et al., 2005), y esta eficacia se ve potenciada por la aplicación de una barrera a la luz (Vilá, 1997), en nuestro caso el polietileno sobre los tocones (Mowatt, 1981). Por ello, esperamos encontrar menor supervivencia y altura de rebrote en ejemplares con tratamientos de tala con aplicación de polietileno en parches con fisonomía de bosque, intermedia en el tratamiento de tala con aplicación de polietileno en los ejemplares localizados en fisonomía de matorral y en los tratamientos de tala localizados en parches de bosque, y mayor en los tratamientos de tala localizados en matorral.

En cuanto a la regeneración por semilla de plantines de L. lucidum, planteamos que sería favorecida por los tratamientos de control mecánico porque generan una reducción de la competencia y mayor disponibilidad de recursos como luz y espacio (Davis et al., 2000), pero este efecto puede quedar enmascarado en los parches de bosque donde la mayor densidad de árboles de distintas especies actúan como dispersores de semillas (Montaldo, 1993; Aragón \& Groom, 2003). Esperamos hallar mayor cantidad de plantines en parches de bosque para ambos tratamientos de control mecánico (tala y tala con polietileno), intermedio en testigo en parches de bosque y testigo en matorral y menor cantidad de plantines en matorral para ambos tratamientos de control mecánico (tala y tala con polietileno).

\section{Materiales y Métodos}

\section{Área de estudio}

El estudio se realizó en la Reserva Natural Vaquerías $\left(31^{\circ} 06^{\prime} \mathrm{LS}, 64^{\circ} 26^{\prime} \mathrm{LW}\right)$, ubicada en las Sierras de Córdoba, centro de Argentina (Fig. 1). El clima es subtropical, con estación invernal seca y verano lluvioso. La temperatura y precipitación anual media son de $15^{\circ} \mathrm{Cy} 750 \mathrm{~mm}$, respectivamente (Capitanelli, 1979). La vegetación pertenece al Chaco Serrano, un bosque xerófilo a subxérofilo cuyas especies dominantes son Schinopsis lorentzii (Griseb.) Engl. y Lithraea molleoides (Vell.) Engl. (Giorgis et al., 2011; Oyarzabal et al., 2018). En 


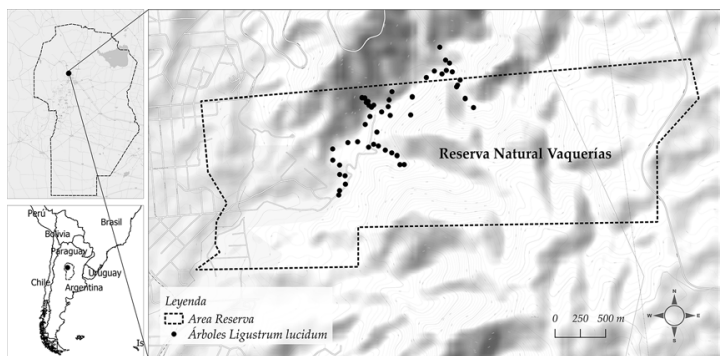

Fig. 1. Ubicación del área de estudio: Reserva Natural Vaquerías y alrededores, en el centro oeste de la provincia de Córdoba, Argentina.

la actualidad se presenta como un mosaico de fisonomías en distintos estados sucesionales, con bosques abiertos a cerrados, matorrales, pastizales y roquedales (Cabido \& Zak, 1999; Gavier-Pizarro \& Bucher, 2004; Giorgis et al., 2017), con distintos grados de invasión de especies no nativas (Giorgis et al., 2011; Salazar et al., 2013). Particularmente, L. lucidum ha alcanzado una extensión aproximada del 7,6\% de la superficie de la reserva (Salazar et al., 2013). Además, se piensa que su expansión ha sido favorecida en sitios donde hubo algún disturbio como la apertura de senderos, la extracción de leña o la deforestación, aunque también está demostrado que $L$. lucidum invade rápidamente sitios sin disturbio (Hoyos et al., 2010; Salazar et al., 2013).

\section{Diseño experimental}

Para evaluar la influencia de la fisonomía de la vegetación en la regeneración por rebrote y semilla tras los tratamientos de control mecánico, seleccionamos 120 individuos jóvenes de $L$. lucidum, entre 3-7 m de altura, la mitad de ellos en parches con fisonomía de bosque y la otra mitad en parches con una fisonomía de matorral. Los bosques estaban codominados por L. molleoides y otras especies leñosas nativas y no nativas, tenían una altura aproximada entre 5 y $10 \mathrm{~m}$ (Salazar et al., 2013) y una cobertura del dosel mayor al $70 \%$, de modo tal que la copa del individuo de $L$. lucidum siempre estaba cubierta (Tabla 1). En los matorrales predominaban las herbáceas y especies leñosas aisladas, tanto nativas como no nativas, cuya altura era inferior a los $2 \mathrm{~m}$ (Salazar et al., 2013), y la cobertura del dosel era menor al $10 \%$, de modo que la copa de L. lucidum no estaba cubierta (Tabla 1). Los sitios fueron similares en altitud, pendiente y orientación (Tabla 1). Los individuos en cada tipo de fisonomía no estuvieron agrupados entre sí (Fig. 1) y fueron asignados al azar a tres grupos de tratamientos de control mecánico: 1) testigo: sin tala ni aplicación de polietileno; 2) tratamiento de tala: consistió en el corte con machete del fuste principal a $10 \mathrm{~cm}$ del suelo, y 3) tratamiento de tala con polietileno: consistió en el corte con machete del fuste

Tabla 1. Características ambientales (media \pm EE) de las parcelas de Ligustrum lucidum localizadas en dos fisonomías de la vegetación (bosque y matorral). n: Número de árboles de L. lucidum y sus correspondientes parcelas.

\begin{tabular}{|c|c|c|c|c|c|c|}
\hline Variable & $n(B)$ & $\mathrm{n}(\mathrm{M})$ & Bosque & Matorral & Wilcoxon & $P$ (2 colas) \\
\hline Cobertura del dosel (\%) & 60 & 60 & $71,8 \pm 13,0$ & $7,8 \pm 7,9$ & 5430 & $<0,0001$ \\
\hline Cobertura de arbustos (\%) & 54 & 52 & $29,1 \pm 14,4$ & $22,6 \pm 14,9$ & 2373 & 0,009 \\
\hline Cobertura de herbáceas (\%) & 54 & 52 & $17,1 \pm 11,8$ & $27,2 \pm 19,3$ & 3207,5 & 0,007 \\
\hline Cobertura de suelo desnudo (\%) & 54 & 52 & $5,7 \pm 8,0$ & $7,6 \pm 9,2$ & 2972,5 & 0,2 \\
\hline Cobertura de hojarasca (\%) & 54 & 52 & $23,9 \pm 14,9$ & $16,7 \pm 10,4$ & 2404,5 & 0,02 \\
\hline Cobertura de roca $(\%)$ & 54 & 52 & $24,4 \pm 17,2$ & $25,9 \pm 21,5$ & 2795,5 & 0,93 \\
\hline Altitud snm (\%) & 60 & 60 & $962,8 \pm 29,6$ & $977,8 \pm 39,0$ & 3298,5 & 0,08 \\
\hline Pendiente $\left({ }^{\circ}\right)$ & 60 & 60 & $37,2 \pm 13,8$ & $40,1 \pm 16,1$ & 3384 & 0,19 \\
\hline $\begin{array}{l}\text { Componente } \mathrm{N}-\mathrm{S} \text { de la } \\
\text { orientación de la pendiente }\end{array}$ & 60 & 60 & $-0,3 \pm 0,7$ & $-0,1 \pm 0,7$ & 3290,5 & 0,07 \\
\hline $\begin{array}{l}\text { Componente E-W de la } \\
\text { orientación de la pendiente }\end{array}$ & 60 & 60 & $-0,02 \pm 0,6$ & $-0,08 \pm 0,8$ & 3780,5 & 0,42 \\
\hline
\end{tabular}




\section{T. A. Valfré-Giorello et al. - Control mecánico de Ligustrum lucidum}

principal a $10 \mathrm{~cm}$ del suelo y aplicación sobre el tocón de una doble capa de polietileno negro, de $200 \mu \mathrm{m}$, de un tamaño aproximado de $50 \times 50 \mathrm{~cm}^{2}$, ajustado a la base con alambre ( 3 tratamientos $\mathrm{x} 2$ fisonomías x 20 individuos $=120$, la cantidad final de individuos varió en los distintos tratamientos debido a pérdidas por vandalismo, quedando un total de 106 individuos). Alrededor de cada individuo adulto se estableció una parcela circular de $4 \mathrm{~m}$ de diámetro, con el objeto de evaluar la regeneración por semilla de L. lucidum. Para cada parcela se estimó visualmente el porcentaje de cobertura del: (1) dosel, (2) vegetación herbácea, (3) arbustos menores a $2 \mathrm{~m}$ de altura, (4) roca, (5) hojarasca y (6) suelo desnudo. Además, se registraron las siguientes variables topográficas: (7) altitud (m), (8) pendiente $\left({ }^{\circ}\right)$, y (9) orientación de la pendiente, considerando los componentes norte-sur y este-oeste, siguiendo la metodología propuesta por Torres et al., (2008).

En la primavera de 2012 se aplicaron por primera vez los tratamientos de tala y tala con polietileno a los individuos adultos seleccionados, y se contabilizaron y removieron manualmente todos los plantines originados de semilla menores a $50 \mathrm{~cm}$ de altura localizados dentro de las parcelas. Transcurridos 6 meses, en otoño de 2013, se repitieron por segunda vez dichos tratamientos. En otoño de 2014 (18 y 12 meses después de la primera y segunda aplicación de los tratamientos, respectivamente) se evaluó la supervivencia, la altura de los rebrotes y de los árboles testigos, y la cantidad de plantines menores a $50 \mathrm{~cm}$ de altura.

Durante la primera aplicación de los tratamientos de corte y la remoción manual de plantines se registró el tiempo que demandó cada tarea y los costos de los insumos utilizados. Para estimar los costos relacionados al esfuerzo humano se consideró el salario de un brigadista forestal, persona que consideramos idónea para realizar esta tarea. Dicho salario es aproximadamente USD 10 hs persona, e incluye insumos (ropa adecuada), viáticos y traslados (información proporcionada por personal de Administración de Parques Nacionales de Argentina).

\section{Análisis de datos}

Todas las variables fueron analizadas mediante modelos lineales empleando como variables predictoras categóricas de efectos fijos el tratamiento de control mecánico (tres niveles), la fisionomía del sitio (dos niveles) y la interacción tratamiento de control $\mathrm{x}$ fisionomía del sitio (seis niveles). La supervivencia de los individuos fue analizada mediante un Modelo Lineal Generalizado con distribución binomial, utilizando el método de verosimilitud penalizada de Firth que permite obtener estimaciones consistentes de los parámetros. El crecimiento de los árboles adultos fue analizado mediante un Modelo Lineal General empleando como variable respuesta el $\log 10$ de la altura. En ambos análisis (supervivencia y altura) se incluyó como covariable a la altura inicial, ya que los individuos en los matorrales presentaban mayor tamaño. Para evaluar la regeneración por semilla en las parcelas bajo distintos tratamientos de control y fisonomías del sitio se empleó un Modelo Lineal Generalizado, asumiendo distribución binomial negativa y empleando el número de plantines por parcela como variable respuesta. Se incluyeron la altura inicial del árbol y la altura inicial al cuadrado como covariables. Se declaró como variable compensatoria (offset) al Log10 del área de la parcela $\left(12,57 \mathrm{~m}^{2}\right)$ para que las comparaciones de medias sean hechas en plantines por $\mathrm{m}^{2}$. Las comparaciones múltiples de medias se realizaron con la prueba LSD de Fisher. Con el fin de interpretar mejor los resultados obtenidos, se compararon las variables de micrositio medidas en los sitios con distinta fisonomía mediante una prueba de Wilcoxon. En todos los análisis se utilizó un nivel de significación de 0,05 y se corroboraron los supuestos correspondientes. Los modelos se ajustaron utilizando la librería de R (R CoreTeam 2016), en la interfaz implementada en el software InfoStat (Di Rienzo et al., 2017 a, b, c).

La eficiencia relativa de los distintos tratamientos en la reducción de la supervivencia y altura de los rebrotes se obtuvo a través de la siguiente ecuación:

$$
\mathrm{IE}=\mathrm{E} / \mathrm{C}
$$

Donde "IE" es el Índice de eficiencia, "E" es la eficacia y "C" es el costo económico de los insumos y el esfuerzo de mano de obra (dólares.árbol ${ }^{-1}$ ). La eficacia (E) se obtuvo de la siguiente manera:

$$
\mathrm{E}=(\mathrm{M} * \mathrm{DA})
$$

Donde "M" es la proporción de mortalidad media y "DA" es la proporción de la disminución en altura media de los rebrotes. 


\section{Resultados}

Luego de dos aplicaciones de los tratamientos, la supervivencia de los árboles asignados a los grupos testigo, tala y tala con polietileno fue de $100 \%$, $95 \%$ y $68 \%$, respectivamente, siendo significativas únicamente las diferencias en el tratamiento de tala con polietileno $(n=106 ; \mathrm{F}=10,9 ; \mathrm{P}<0,0001$; Fig. 2A). No se encontró un efecto de la fisonomía del sitio sobre la supervivencia $(\mathrm{F}=-1,7 ; \mathrm{P}=1)$ y el modelo sin interacción tuvo mejor ajuste. Por su parte, la altura inicial de los árboles estuvo positivamente relacionada con la supervivencia $(\mathrm{F}=$ 7,2; $\mathrm{P}=0,009)$.

La altura alcanzada por los árboles asignados a los grupos testigo, tala y tala con polietileno fue de 509,$1 ; 113,0$ y $24,4 \mathrm{~cm}$, respectivamente, siendo significativa la diferencia entre todos los tratamientos de control mecánico $(n=93 ; \mathrm{F}=240 ; \mathrm{P}<0,0001)$. En otras palabras, la altura de los árboles talados se redujo un $78 \%$ en relación a los árboles testigos, mientras que en el tratamiento de tala con polietileno se redujo un $95 \%$ en comparación con los testigos (Fig. 2B). No se encontró un efecto de la fisonomía del sitio sobre la altura de los rebrotes y testigos $(\mathrm{F}=$ $0,06 ; \mathrm{P}=0,8)$, ni efecto de la interacción tratamiento $\mathrm{x}$ fisonomía del sitio $(\mathrm{F}=0,7 ; \mathrm{P}=0,5)$. Mientras que la altura de los rebrotes y del árbol testigo estuvo positivamente relacionada con la altura inicial de los árboles $(\mathrm{F}=13,2 ; \mathrm{P}<0,001)$.
En cuanto a la regeneración por semilla, al inicio y antes de la aplicación de los tratamientos de control mecánico removimos 2417 plantines $\left(1,81\right.$ plantines. $\left.\mathrm{m}^{-2}\right)$, mientras que al final del estudio y luego de la aplicación de los tratamientos en dos oportunidades, removimos 2794 plantines $\left(2,1\right.$ plantines. $\left.\mathrm{m}^{-2}\right)$. La densidad de plantines fue explicada por la interacción fisonomía del sitio $\mathrm{x}$ tratamiento $(\mathrm{n}=106 ; \mathrm{F}=3,29 ; \mathrm{P}=0,04)$, y cada uno de dichos factores también tuvo un efecto significativo (fisonomía del sitio: $\mathrm{F}=7,96 ; \mathrm{P}=$ 0,006; tratamiento: $\mathrm{F}=4,77 ; \mathrm{P}=0,011)$. En los bosques, la densidad de plantines fue menor en los tratamientos de tala y tala con polietileno que en el testigo, mientras que en los matorrales la densidad de plantines fue menor en el tratamiento de tala con polietileno que en el tratamiento de tala, sin diferencias significativas entre estos tratamientos y el testigo (Fig. 2C). En promedio, la densidad de plantines fue aproximadamente $300 \%$ mayor en bosques que en matorrales (3,34 y 0,81 plantines. $\mathrm{m}^{-2}$, respectivamente), aunque este valor estuvo mayormente influido por el tratamiento testigo. Por su parte, la altura inicial de los árboles $(\mathrm{F}=5,22 ; \mathrm{P}=0,024)$ y la altura al cuadrado $(\mathrm{F}=5,5 ; \mathrm{P}=0,021)$, estuvieron positivamente relacionadas a la densidad de plantines, hecho que evidencia que en las parcelas con árboles de tamaño intermedio regeneraron la mayor cantidad de plantines.
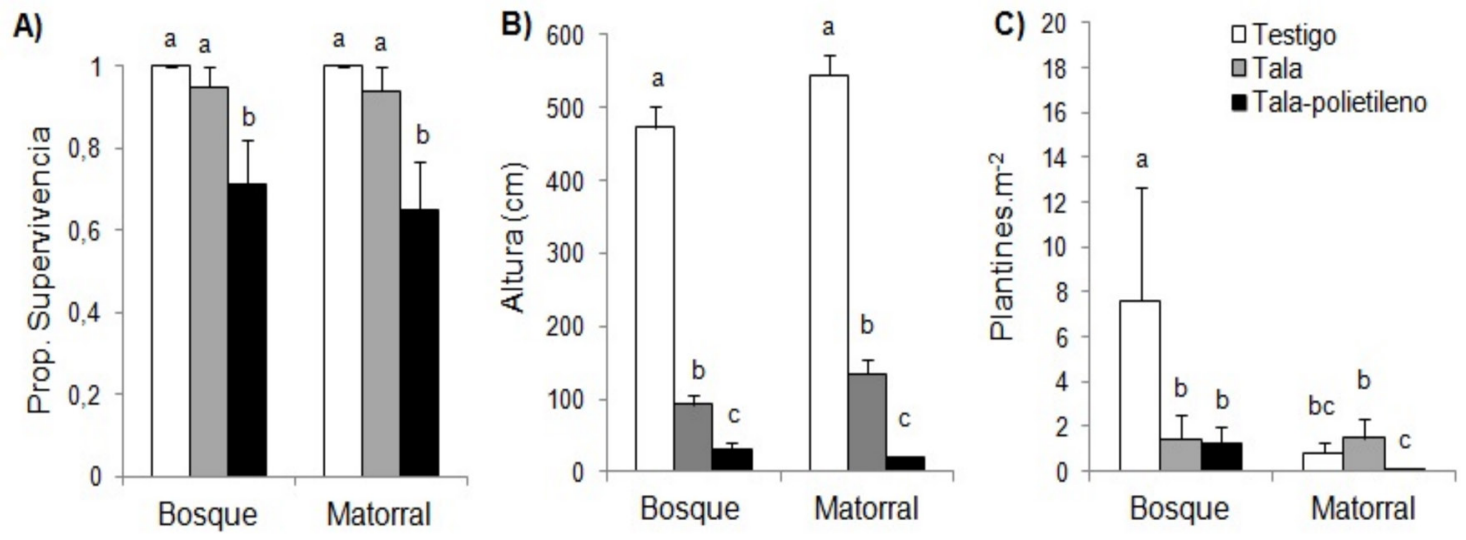

Fig. 2. Media \pm EE de la A: supervivencia, B: altura de rebrotes $(\mathrm{cm})$, y C: densidad de plantines. $\mathrm{m}^{-2}$ de Ligustrum lucidum en dos fisonomías de la vegetación (bosque y matorral), tras 12 meses y dos aplicaciones de los tratamientos de control mecánico (testigo, tala y tala-polietileno). Letras diferentes sobre las barras indican diferencias significativas a un nivel de $P<0,05$. 


\section{T. A. Valfré-Giorello et al. - Control mecánico de Ligustrum lucidum}

Las parcelas en bosque y matorrales se diferenciaron principalmente en el porcentaje de cobertura del dosel, siendo aproximadamente del $70 \%$ en bosque y del $7 \%$ en matorrales. Las parcelas en matorrales se hallaban en una matriz de pastizal, mientras que en bosque predominó la presencia de arbustos menores a $2 \mathrm{~m}$ de altura y hojarasca. La altitud snm, pendiente y orientación no mostraron diferencias entre parcelas de bosque y matorrales (Tabla 1).

En cuanto a los costos estimados durante el control mecánico, la tala demandó 4,17 min. persona.árbol ${ }^{-1}$, mientras que la tala con polietileno demandó 7,5 min.persona.árbol ${ }^{-1}$, lo cual se traslada a un costo monetario en USD de 0,7 y 1,74 , respectivamente, incluyendo en el segundo caso el costo de mano de obra, polietileno y alambre. De esta manera, se observa que el costo del tratamiento de tala con polietileno es $150 \%$ veces mayor que la tala. Por otra parte, la cantidad de plantines encontrados varió desde 0 hasta 80 plantines. $\mathrm{m}^{-2}$ y el tiempo total empleado en esta actividad fue de 18,75 hs, casi 1 min.persona.m ${ }^{-2}$. Como no encontramos diferencias en el tiempo para remover plantines entre tratamientos, no fue incluida esta sección en los cálculos de la eficiencia relativa. No se consideró el tiempo que requirió la movilización desde un árbol hasta otro durante la aplicación de los tratamientos.

En este sentido, se obtuvo una $\mathrm{E}$ del 0,04 para tala $(0,05$ de mortalidad x 0,78 de disminución en altura de rebrote -ver resultados correspondientes-) y del 0,31 para tala con polietileno $(0,32 \mathrm{de}$ mortalidad x 0,95 de disminución en altura de rebrote -ver resultados correspondientes-), es decir que la tala con polietileno fue aproximadamente $650 \%$ más eficaz que la tala. Si incluimos el costo económico y obtenemos el IE, la diferencia entre los tratamientos se reduce pero continúa siendo más eficiente (200\%) la tala con polietileno (Tabla 2).

Tabla 2. Tiempo, costos, mortalidad (M), disminución de la altura (DA), eficacia (E) e índice de eficiencia

(IE) de los tratamientos de control mecánico (tala y tala con polietileno) sobre Ligustrum lucidum, tras

12 meses y dos aplicaciones. Los valores (media \pm EE) están calculados por árbol y por persona. Los

costos de insumos corresponden al polietileno negro y alambre. Los costos de esfuerzo de mano de obra se estimaron tasando la hora de trabajo de un brigadista forestal asalariado. Hora: USD 10. Eficacia $E=M * D A$. Índice de Eficiencia IE = E/Costos totales.

\begin{tabular}{|lcc|}
\hline \multicolumn{1}{|c}{ Variable } & Tala & Tala con Polietileno \\
\hline Tiempo (minutos) & 4,17 & 7,5 \\
Costos insumos (USD) & 0 & 0,49 \\
Costo esfuerzo (USD) & $0,7 \pm 0,07$ & $1,25 \pm 0,10$ \\
Proporción de mortalidad (M) & 0,05 & 0,32 \\
Proporción disminución altura (DA) & 0,78 & 0,95 \\
Eficacia (E) & 0,04 & 0,31 \\
Índice de Eficiencia (IE) & 0,06 & 0,18 \\
\hline
\end{tabular}

\section{Discusión}

Este estudio constituye, a nuestro mejor entender, el primer reporte para el Bosque Chaqueño Serrano sobre la eficiencia de técnicas de control mecánico de leñosas no nativas, incluyendo costos y esfuerzo de aplicación. En Sudamérica, los estudios publicados sobre acciones de control, representan tan solo un $1 \%$ de la literatura científica sobre invasiones biológicas (Kettenring \& Adams, 2011), por lo cual los resultados aquí obtenidos son muy importantes como primera etapa en la elaboración de una estrategia de manejo de plantas leñosas no nativas (Epanchin-Niell \& Hastings, 2010; Kettenring \& Adams, 2011). Estudios previos han evaluado la eficacia de la aplicación de herbicidas para el control de especies del género Ligustrum (Starr et al., 2003; DiTomaso et al., 2013), sin 
embargo la optimización del control a través de técnicas mecánicas es fundamental en un contexto de creciente rechazo social al uso de herbicidas (Little et al., 2006; McCarthy et al., 2011).

Tal como esperábamos, la aplicación de los tratamientos de control mecánico redujo la supervivencia y la altura de rebrote en la medida que disminuyó la incidencia de luz. De esta manera, el tratamiento más efectivo resultó ser la tala con aplicación de polietileno, que redujo la supervivencia un $32 \%$ y la altura de rebrote en un $95 \%$, en comparación con los testigos no tratados. La aplicación de la tala no redujo de manera significativa la supervivencia, y los rebrotes alcanzaron un cuarto de la altura en relación a los testigos. Esta alta capacidad de recuperación por rebrote luego de la remoción de la biomasa aérea ha sido reportada por otros trabajos (Starr et al., 2003; Lichstein et al., 2004; DiTomaso et al.2013), aunque no en todas las condiciones ya que después del fuego la supervivencia por rebrote es menor al $25 \%$ y solo los individuos de gran tamaño pueden rebrotar desde el tallo (Torres et al., 2014; Herrero et al., 2016). Un estudio previo había señalado a la tala con aplicación de polietileno como un tratamiento eficaz en el control de L. lucidum, mientras sea cubierta toda la parte aérea remanente; sin embargo se trató de resultados obtenidos en el corto plazo y luego de la aplicación de un único corte (Mowatt, 1981).

La fisonomía del sitio no provocó diferencias en la supervivencia y altura de los rebrotes. Esto indicaría que en esta etapa del ciclo de vida, y debido a la capacidad que tiene la especie de tolerar una amplia gama de condiciones ambientales (Aragón \& Groom, 2003; Hoyos et al., 2010; Maddox et al., 2010), las diferencias entre fisonomías resulten poco limitantes en la supervivencia y crecimiento de los rebrotes. En este sentido, y con resultados opuestos, se observó que la especie no nativa Gleditsia triacanthos, tuvo menor tasa de crecimiento radial del leño en parches con abundante cobertura de bosque que en sitios más abiertos (Fraschina, 2014), asociando dichas respuestas a las características heliófilas de la planta.

Por otra parte, como el tamaño de los árboles condiciona la capacidad de rebrote, esto refuerza la importancia de iniciar las acciones de manejo en una etapa temprana de invasión y crecimiento
(Rejmánek \& Pitcairn, 2002), por ejemplo sería conveniente aplicar los tratamientos antes que los individuos sobrepasen la canopia del bosque nativo (aproximadamente $10 \mathrm{~m}$ ), ya que en estas instancias parece tener su mayor capacidad de dispersión (Hoyos et al., 2010; Gavier-Pizarro et al., 2012) y generar las mayores pérdidas en biodiversidad (Hoyos et al., 2010). Dada la relación entre tamaño de los árboles y edad (Edad del árbol $=4,11+$ 0,9267 DAP; Gavier-Pizarro et al., 2012), podemos estimar que la mayoría de los árboles de este estudio son ejemplares jóvenes (entre 10-20 años). Sería interesante determinar cómo es la relación entre la edad y la capacidad de rebrote y si a cierta edad esta capacidad de rebrote disminuye.

La regeneración de plantines por semilla mostró una clara asociación a la fisonomía de bosque y a los tratamientos testigos, es decir a los que conservaban la copa del árbol. Esto puede ser el resultado de muchos mecanismos que actúan conjuntamente. Por un lado, las condiciones microambientales de luz, temperatura y humedad en los parches de bosque pueden ser más propicias y le permitan mayor germinación y supervivencia que en los matorrales. Esto fue observado por Tecco et al., (2006) para esta misma especie, quienes hallaron mayor regeneración en los arbustos de copas más densa y dicha cobertura estuvo asociada a un gradiente de temperatura, humedad y nutrientes más favorable para el establecimiento, como así también mayor protección frente al ganado. Otro de los mecanismos puede estar relacionado a la mayor dispersión de semillas en los parches de bosque, ya sea a través de otros individuos semilleros de L. lucidum en los alrededores de los árboles estudiados (Ferreras et al., 2015) o a la presencia de otras especies arbóreas que actúen como percha (Montaldo, 1993; Aragón \& Groom, 2003). Si bien la densidad de otros individuos de L. lucidum no fue registrada, en los parches de bosque había mayor presencia que en los matorrales (Salazar et al., 2013), lo cual pudo haber generado mayor lluvia de semillas (Ferreras et al., 2008; Ferreras et al., 2015). Además, observamos que la cantidad de plantines luego de dos extracciones fue similar a la cantidad encontrada antes de la aplicación de los tratamientos. Al tratarse de una especie que no forma banco de semillas por más de un año (Panetta, 2000), la presión de propágulos es tan elevada que logra rápidamente restituir la densidad (Ferreras et 


\section{T. A. Valfré-Giorello et al. - Control mecánico de Ligustrum lucidum}

al., 2008; Ferreras et al., 2015). Por otro lado, sería interesante considerar en futuras investigaciones en qué magnitud la aplicación de estos tratamientos de control mecánico afectan la regeneración y la supervivencia de las especies nativas.

Respecto al costo y esfuerzo de los tratamientos aplicados, la tala con polietileno insumió aproximadamente $150 \%$ más de los costos monetarios y de tiempo que la tala, pero esto fue compensado por la mayor eficacia $(650 \%)$ y eficiencia $(200 \%)$ de este tratamiento. Cabe destacar que los costos estimados en el presente trabajo pueden variar debido a factores como el tamaño del árbol, la topografía del área invadida y la densidad de individuos. El DAP de los árboles talados en el presente estudio varió entre $3 \mathrm{~cm}$ y $20 \mathrm{~cm}$, pero L. lucidum puede alcanzar diámetros superiores (p.e. $63 \mathrm{~cm}$ Gavier-Pizarro et al., 2012), por lo cual árboles de mayor porte pueden requerir mayor esfuerzo y el uso de otras herramientas, como motosierra, que facilitan la tala y disminuyen el tiempo de trabajo pero implican mayores costos. Además, en el caso de áreas invadidas en regiones montañosas la pendiente del terreno puede ser muy variable, dificultando el acceso a los focos de invasión y el traslado del personal desde un árbol a otro. La densidad de individuos en las áreas invadidas también condiciona el esfuerzo y los costos de control, por ejemplo en áreas muy invadidas por $L$. lucidum en el Bosque Serrano se ha registrado una densidad de 3300 individuos/ha con un DAP $>2,5$ $\mathrm{cm}$ (Hoyos et al., 2010), lo que significaría un costo de US\$2310 para el tratamiento de tala y US\$5742 para el tratamiento de tala con polietileno, valores extremadamente altos para llevarlos a la práctica. De esta manera, los tratamientos serían factibles de realizar en áreas reducidas y con el uso de otras herramientas, como motosierra.

\section{Conclusiones}

Frente a la situación de control mecánico de esta especie, recomendamos la aplicación del tratamiento de la tala con polietileno debido a la mayor eficiencia, en comparación con el tratamiento de tala. Para aumentar el efecto de aislamiento y la durabilidad del material, sugerimos utilizar un polietileno con un grosor mayor a $200 \mu \mathrm{m}$, y que durante el proceso de colocación, se cubra completamente el tocón y el suelo a su alrededor. A los fines de evitar el vandalismo, recomendamos señalizar con carteles informativos las inmediaciones de los sitios donde se aplique este tratamiento. Por otro lado, a causa de los altos costos monetarios, el tratamiento de tala con polietileno resultará más conveniente aplicarlo en árboles que se encuentren alejados y sea difícil volver a controlarlos, para así evitar nuevos focos de invasión, mientras que el tratamiento de tala sería recomendable sólo en los casos que sea posible reaplicar el corte para evitar la rápida recuperación de la biomasa. En cuanto a la remoción manual de plantines sugerimos practicarla en matorrales o en áreas con baja cobertura de L. lucidum, ya que en esos sitios la presión de semillas suele ser más baja que en bosques y de este modo no se restituirá tan rápidamente la densidad de plantines.

\section{Contribución DE los AUtORES}

DR y TAVG: Diseño de la investigación. FRB: Adquisición de financiamiento. TAVG: Ejecución de la metodología. TAVG y RCT: Análisis de datos. TAVG y RCT: Escritura del manuscrito. DR y FRB: Administración del proyecto y supervisión.

\section{Agradecimientos}

Agradecemos al Lic. G. González por sus aportes en los análisis estadísticos. Al Dr. L. Agost por su colaboración en la confección del mapa. A los Guardaparques de la Reserva Natural Vaquerías J. Piedrabuena y E. Galli por su ayuda a campo. Este trabajo forma parte de la Tesis de Maestría en Manejo de Vida Silvestre de la primera autora. Fue realizado con financiación de la Universidad Nacional de Córdoba, a través de la Reserva Natural Vaquerías, y con el Programa de Maestría en Manejo de Vida Silvestre, el cual cuenta con el apoyo del Servicio de Pesca y Vida Silvestre de los Estados Unidos de América (U.S.F.W.S).

\section{Bibliografía}

ARAGÓN, R. \& M. GROOM. 2003. Invasion by Ligustrum lucidum (Oleaceae) in NW Argentina: early stage characteristics in different habitat types. Rev. Biol. Trop. 51: 59-70. 
ARAGÓN, R., L. MONTTI, M. M. AYUP \& R. FERNÁNDEZ. 2014. Exotic species as modifiers of ecosystem processes: Litter decomposition in native and invaded secondary forests of NW Argentina. Acta Oecol. 54: 21-28.

http://dx.doi.org/10.1016/j.actao.2013.03.007

BURNHAM, K. M. \& T. D. LEE. 2010. Canopy gaps facilitate establishment, growth, and reproduction of invasive Frangula alnus in a Tsuga canadensis dominated forest. Biol. Invasions 12: 1509-1520. https://doi:10.1007/s10530-009-9563-8

CABIDO, M. R. \& M. ZAK. 1999. Vegetación del Norte de Córdoba. Instituto Multidisciplinario Biología Vegetal. Universidad Nacional de Córdoba y CONICET. Córdoba, Argentina.

CAPITANELli, R. G. 1979. Clima. En: VÁZQUEZ, J. B., R. A. MIATELLO \& M. E. ROQUÉ (eds.), Geografía física de la Provincia de Córdoba, pp. 45138. Boldt, Buenos Aires.

DAVIS, M. A., J. P. GRIME \& K. THOMPSON. 2000. Fluctuating resources in plant communities: a general theory of invasibility. J. Ecol. 88: 528-534. https://doi.org/10.1046/j.1365-2745.2000.00473.x

DI RIENZO, J. A., F. CASANOVES, M. G. BALZARINI, L. GONZALEZ, M. TABLADA \& C. W. ROBLEDO. 2017a. InfoStat. Córdoba, Argentina. Grupo InfoStat. Disponible en: http:// www.infostat.com.ar

DI RIENZO, J. A., E. MACCHIAVELLI \& F. CASANOVES. 2017b. Modelos lineales generalizados mixtos: aplicaciones en InfoStat. Grupo InfoStat, Córdoba.

DI RIENZO, J. A., E. MACCHIAVELLI \& F. CASANOVES. 2017c. Modelos lineales mixtos: aplicaciones en InfoStat. Grupo InfoStat, Córdoba.

DITOMASO, J. M., G. B. KYSER, S. R. ONETO, R. G. WILSON, S. B. ORLOFF, L. W. ANDERSON \& C. RANSOM. 2013. Weed control in natural areas in the western United States. Weed Research and Information Center, University of California.

EPANCHIN-NIELL, R. S. \& A. HASTINGS. 2010. Controlling established invaders: integrating economics and spread dynamics to determine optimal management. Ecol. Lett. 13: 528-541. http://dx.doi.org/10.1111/j.1461-0248.2010.01440.x

FERRERAS, A. E., C. TORRES \& L. GALETTO. 2008. Fruit removal of an invasive exotic species (Ligustrum lucidum) in a fragmented landscape. $J$. Arid Environ. 72: 1573-1580.

https://doi.org/10.1016/j.jaridenv.2008.03.015

FERRERAS, A. E., M. A. GIORGIS, P. A. TECCO, M. R. CABIDO, \& G. FUNES. 2015. Impact of Ligustrum lucidum on the soil seed bank in invaded subtropical seasonally dry woodlands (Córdoba, Argentina). Biol. Invasions 17: 3547-3561. http://dx.doi.org/10.1007/s10530-015-0977-1
FRASCHINA, L. 2014. Efecto de la cobertura de bosque nativo sobre la tasa de crecimiento de la especie invasora Gleditsia triacanthos L. en la Reserva Natural Vaquerías, Córdoba. Tesina de grado. Universidad Nacional de Córdoba.

GAVIER-PIZARRO, G. I. \& E. BUCHER. 2004. Deforestación de las Sierras Chicas de Córdoba (Argentina) en el período 1970-1997. Boletín de la Academia Nacional de Ciencias, Córdoba, Argentina 101: 3-27.

GAVIER-PIZARRO, G. I., T. KUEMMERLE, L. E. HOYOS, S. I. STEWART, C. D. HUEBNER, N. S. KEULER \& V. C. RADELOFF. 2012. Monitoring the invasion of an exotic tree (Ligustrum lucidum) from 1983 to 2006 with Landsat TM/ETM+ satellite data and Support Vector Machines in Córdoba, Argentina. Remote Sens. Environ. 122: 134-145. https://doi.org/10.1016/j.rse.2011.09.023

GIORGIS, M. A., P. A. TECCO, A. M. CINGOLANI, D. RENISON, P. MARCORA \& V. PAIARO. 2011. Factors associated with woody alien species distribution in a newly invaded mountain system of central Argentina. Biol. Invasions 13: 1423-1434. https://doi.org/10.1007/s10530-010-9900-y

GIORGIS, M. A, A. M. CINGOLANI, D. E. GURVICH, P. A. TECCO, J. CHIAPELLA, F. E. CHIARINI \& M. R. CABIDO. 2017. Changes in floristic composition and physiognomy are decoupled along elevation gradients in central Argentina. Appl. Veget. Sci. 20: 558-571. https://doi.org/10.1111/avsc.12324

GURVICH, D. E., L. ENRICO \& A. M. CINGOLANI. 2005. Linking plant functional traits with postfire sprouting vigour in woody species in central Argentina. Austral Ecol. 30: 789-796.

https://doi.org/10.1111/j.1442-9993.2005.01522.x

HERRERO, M. L., R. C. TORRES \& D. RENISON. 2016. Do wildfires promote woody species invasion in a fire-adapted ecosystem? Post-fire resprouting of native and non-native woody plants in central Argentina. Environ. Manage. 57: 308-317. http://dx.doi.org/10.1007/s00267-015-0616-8

HOYOS, L. E., G. I. GAVIER-PIZARRO, T. KUEMMERLE, E. H. BUCHER, V. C. RADELOFF \& P. A. TECCO. 2010. Invasion of glossy privet (Ligustrum lucidum) and native forest loss in the Sierras Chicas of Córdoba, Argentina. Biol. Invasions 12: 3261-3275. https://doi.org/10.1007/s10530-010-9720-0

KAYS, J. S \& C. D. CANHAM. 1991. Effects of time and frequency of cutting on hardwood root reserves and sprout growth. Forest Sci. 37: 524-539.

KETTENRING, K. M. \& C. R ADAMS. 2011. Lessons learned from invasive plant control experiments: a systematic review and metaanalysis. J. Appl. Ecol. 48: 970-979.

https://doi.org/10.1111/j.1365-2664.2011.01979.x 


\section{T. A. Valfré-Giorello et al. - Control mecánico de Ligustrum lucidum}

KING, D. A., S. J. DAVIES, M. N. NURSUPARDI \& S. TAN. 2005. Tree growth is related to light interception and wood density in two mixed dipterocarp forests of Malaysia. Funct. Ecol. 19: 445-453. https://doi.org/10.1111/j.1365-2435.2005.00982.x

LEWIS, J. P., S. NOETINGER, D. E. PRADO \& I. M. BARBERIS. 2004. Los remanentes de bosques del Espinal en el este de la provincia de Córdoba. Revista Agromensajes 13: 23-27.

LICHSTEIN, J. J., H. R. GRAU, R. ARAGÓN. 2004. Recruitment limitation in secondary forests dominated by an exotic tree. J. Veg. Sci. 15: 721-728. https://doi.org/10.1111/j.1654-1103.2004.tb02314.x

LITTLE, K. M., I. WILLOUGHBY, R. G. WAGNER, P. ADAMS, H. FROCHOT, J. GAVA \& R. P. WEI. 2006. Towards reduced herbicide use in forest vegetation management. Southern Africa Forestry Journal 207: 63-79. https://doi.org/10.2989/10295920609505254

MADDOX, V., J. BYRD \& B. SERVISS. 2010. Identification and control of invasive Privets (Ligustrum spp.) in the Middle Southern United States. Invas. Plant Sci. Mana. 3: 482-488. https://doi.org/10.1614/IPSM-D-09-00060.1

MCCARTHY, N., N. S. BENTSEN, I. WILLOUGHBY \& P. BALANDIER. 2011. The state of forest vegetation management in Europe in the 21st century. Eur. J. For. Res. 130: 7-16. https://doi.org/10.1007/s10342-010-0429-5

MONTALDO, N. H. 1993. Dispersión por aves y éxito reproductivo de dos especies de Ligustrum (Oleaceae) en un relicto de selva subtropical en la Argentina. Rev. Chil. Hist. Nat. 66: 75-85.

MOWATT, J. 1981. Control of large-leaved privet (Ligustrum lucidum) and small leaved privet (Ligustrum sinense) in urban bushland. Proceedings of the Australasian Weeds Conference 1: 165-168.

OYARZABAL, M., J. CLAVIJO, L. OAKLEY, F. BIGANZOLI, P. TOGNETTI, I. BARBERIS, H. M. MATURO, R. ARAGÓN, P. I. CAMPANELLO, D. PRADO, M. OESTERHELD \& R. J. C. LEÓN. 2018. Unidades de vegetación de la Argentina. Ecol. Aust. 28: 040-063. https://doi.org/10.25260/EA.18.28.1.0.399

PACKHAM, J. R., D. J. L. HARDING, G. M. HILTON \& R. A. STUTTARD. 1992. Functional ecology of woodlands and forests. Chapman and Hall, London

PANETTA, F. D. 2000. Fates of fruits and seeds of Ligustrum lucidum WT Ait. and L. sinense Lour. maintained under natural rainfall or irrigation. Aust. J. Bot. 48: 701-706. https://doi.org/10.1071/BT00005

PATCH, N. L., D. GEESING \& P. FELKER. 1998. Suppression of resprouting in pruned mesquite (Prosopis glandulosa var. glandulosa) saplings with chemical or physical barrier treatments. For. Ecol. Manage. 112: 23-29.

https://doi.org/10.1016/S0378-1127(98)00303-X
PEJCHAR, L. \& H. A. MOONEY. 2009. Invasive species, ecosystem services and human well-being. Trends Ecol. Evol. 24: 497-504. http://dx.doi.org/10.1016/j.tree.2009.03.016

PIMENTEL, D., S. MCNAIR, J. JANECKA, J. WIGHTMAN, C. SIMMONDS, C. O'CONNELL, E. WONG, L. RUSSEL, J. ZERN, T. AQUINO \& T. TSOMONDO. 2001. Economic and environmental threats of alien plant, animal, and microbe invasions. Agric. Ecosyst. Environ. 84: 1-20. http://dx.doi.org/10.1016/S0167-8809(00)00178-X

PYŠEK, P. \& D. M. RICHARDSON. 2010. Invasive Species, Environmental Change and Management, and Health. Ann. Rev. Env. Resour. 35: 25-55. https://doi:10.1146/annurev-environ-033009-095548

R DEVELOPMENT CORE TEAM. 2016. R: A language and environment for statistical computing. $\mathrm{R}$ Foundation for Statistical Computing, Vienna, Austria. ISBN 3-900051-07-0. [Online] Disponible en: http://www.R-project.org

REJMÁNEK, M. \& M. J. PITCAIRN. 2002. When is eradication of exotic pest plants a realistic goal? In: VEITCH, C. R. \& M. N. CLOUT (eds.), Turning the tide: the eradication of invasive species, pp. 249253. IUCN SSC Invasive Species Specialist Group. IUCN, Gland, Switzerland and Cambridge, UK.

RICHARDSON, D. M. \& M. REJMÁNEK. 2011. Trees and shrubs as invasive alien species-a global review. Divers. Distrib. 17: 788-809. https://doi.org/10.1111/j.1472-4642.2011.00782.x

SALAZAR, J., F. BARRI \& G. CARDOZO. 2013. Distribución espacial y tasa de invasión de flora exótica en la Reserva Natural de VaqueríasProvincia de Córdoba (Argentina). Quad. Bot. Amb. Appl. 24: 3-12.

SANHUEZA, C. \& S. M. ZALBA. 2012. Experimental control of Spanish broom (Spartium junceum) invading natural grasslands. Manag. Biol. Invasion. 3: $97-104$.

http://dx.doi.org/10.3391/mbi.2012.3.2.04

SIMBERLOFF, D., J. L. MARTIN, P. GENOVESI, V. MARIS, D. A. WARDLE, J. ARONSON, F. COURCHAMP, B. GALIL, E. GARCÍABERTHOU, M. PASCAL, P. PYŠEK, R. SOUSA, E. TABACCHI \& M. VILA. 2013. Impacts of biological invasions: what's what and the way forward. Trends Ecol. Evol. 28: 58-66. https://doi.org/10.1016/j.tree.2012.07.013

STARR, F., K. STARR \& L. LOOPE. 2003. Ligustrum spp. Privet Oleaceae. United States Geological Survey-Biological Resources Division Haleakala Field Station, Maui, Hawaii. [Online] Disponible en: http://hear.its.hawaii.edu/starr/hiplants/reports/pdf/ ligustrum_spp.pdf. [Acceso: 10 Noviembre 2018].

TAIZ, L. \& E. ZEIGER. 2010. Plant Physiology. 5th ed. Sunderland, MA: Sinauer Associates. 


\section{Bol. Soc. Argent. Bot. 54 (1) 2019}

TECCO, P. A., D. E. GURVICH, S. DIAZ, N. PÉREZHARGUINDEGUY \& M. R. CABIDO. 2006. Positive interaction between invasive plants: the influence of Pyracantha angustifolia on the recruitment of native and exotic woody species. Austral Ecol. 31: 293-300.

https://doi.org/10.1111/j.1442-9993.2006.01557.x

TORRES, R. C., D. RENISON, I. HENSEN, R. SUAREZ, L. ENRICO. 2008. Polylepis australis' regeneration niche in relation to seed dispersal, site characteristics and livestock density. For. Ecol. Manage. 254: 255260. https://doi.org/10.1016/j.foreco.2007.08.007

TORRES, R. C., M. A. GIORGIS, C. TRILLO, L. VOLKMANN, P. DEMAIO, J. HEREDIA \& D. RENISON. 2014. Post-fire recovery occurs overwhelmingly by resprouting in the Chaco Serrano forest of Central Argentina. Austral Ecol. 39: 346354. https://doi.org/10.1111/aec.12084

TU, M., C. HURD \& J. M. RANDALL. 2001. Weed Control Methods Handbook: Tools and Techniques for Use in Natural Areas. The Nature Conservancy. [Online] Disponible en: https:// www.invasive.org/gist/handbook.html [Acceso: 10 Noviembre 2018].

VILÁ, M. 1997. Effect of root competition and shading on resprouting dynamics or Erica multiflora L. J. Veg. Sci. 8: 71-80. https://doi.org/10.2307/3237244

ZALBA, S. M. \& C. B. VILLAMIL. 2002. Woody plant invasion in relictual grasslands. Biol. Invasions 4: 55-72. https://doi.org/10.1023/A:1020532609792 